\title{
Eribulin in combination with bevacizumab as second-line treatment for HER2-negative metastatic breast cancer progressing after first-line therapy with paclitaxel and bevacizumab: a multicenter, phase II, single arm trial (GIM11-BERGI)
}

\section{CARMINE DE ANGELIS}

Universita degli Studi di Napoli Federico II https://orcid.org/0000-0003-1158-4630

\section{Dario Bruzzese}

Universita degli Studi di Napoli Federico II Dipartimento di Sanita Pubblica

Antonio Bernardo

Fondazione Salvatore Maugeri Istituto Scientifico di Pavia Via Maugeri

Editta Baldini

Department of Oncology, San Luca Hospital, Lucca

\section{Luigi Leo}

AORN Ospedali dei Colli

\section{Alessandra Fabi}

Medical Oncology 1, IRCCS Regina Elena National Cancer Institute, Rome

\section{Teresa Gamucci}

Medical Oncology Unit, ASL Frosinone, Frosinone

\section{Pietro De Placido}

Universita degli Studi di Napoli Federico II Dipartimento di Medicina Clinica e Chirurgia

\section{Francesca Poggio}

Ospedale Policlinico San Martino

\section{Stefania Russo}

Azienda Sanitaria Universitaria Integrata di Udine

\section{Valeria Forestieri}

Universita degli Studi di Napoli Federico II Dipartimento di Medicina Clinica e Chirurgia

\section{Rossella Lauria}

Universita degli Studi di Napoli Federico II Dipartimento di Medicina Clinica e Chirurgia Irene De Santo

Universita degli Studi di Napoli Federico II Dipartimento di Medicina Clinica e Chirurgia Andrea Michelotti 


\section{Lucia Del Mastro}

Ospedale Policlinico San Martino

\section{Michelino De Laurentiis}

Istituto Nazionale Tumori IRCCS Fondazione Pascale

\section{Mario Giuliano ( $\sim$ m.giuliano@unina.it)}

Universita degli Studi di Napoli Federico II https://orcid.org/0000-0003-0835-3153

\section{Sabino De Placido}

Universita degli Studi di Napoli Federico II Dipartimento di Medicina Clinica e Chirurgia

\section{Grazia Arpino}

Universita degli Studi di Napoli Federico II Dipartimento di Medicina Clinica e Chirurgia

\section{Research article}

Keywords: Eribulin, bevacizumab, HER2-negative, metastatic breast cancer

Posted Date: August 12th, 2020

DOl: https://doi.org/10.21203/rs.3.rs-57677/v1

License: (c) (1) This work is licensed under a Creative Commons Attribution 4.0 International License. Read Full License

Version of Record: A version of this preprint was published at ESMO Open on April 1st, 2021. See the published version at https://doi.org/10.1016/j.esmoop.2021.100054. 


\section{Abstract}

Background: Currently, there is no standard second-line chemotherapy-based treatment for patients with HER2-negative metastatic breast cancer (MBC). Continued VEGF inhibition with bevacizumab is an option in patients progressing to first-line bevacizumab and chemotherapy. Eribulin is a non-taxane microtubule dynamics inhibitor with a unique mechanism of action that has been shown to exert beneficial effects in the tumor microenvironment and on neoangiogenesis. We evaluated the efficacy and safety of eribulin plus bevacizumab in a novel second-line chemotherapy scheme in patients progressing after first-line paclitaxel and bevacizumab.

Methods: This is a multicenter, single-arm, Simon's two-stage, phase II study. Patients with HER2-negative MBC progressing to paclitaxel and bevacizumab received eribulin $\left(1.23 \mathrm{mg} / \mathrm{m}^{2}\right.$ intravenously on days 1 and 8 of each 21-day cycle) plus bevacizumab (10 mg/kg every 2 weeks or $15 \mathrm{mg} / \mathrm{kg}$ every 3 weeks intravenously), as second-line chemotherapy. The primary endpoint was the overall response rate, considered as the sum of partial (PR) and complete response (CR) based on the best overall response (BORR).

Results: Fifty-eight (95.1\%) of the 61 patients enrolled in the study were evaluable for efficacy. The BORR was $24.6 \%$ (95\% confidence intervals [CI], $14.5 \%$ to $37.3 \%$ ). The CBR (i.e. the CR + PR + SD response that persisted for more than 24 weeks) was $32.8 \%(95 \% \mathrm{Cl}, 21.3 \%$ to $46.0 \%)$. The median progression-free survival was 6.2 months ( $95 \% \mathrm{Cl}, 4.0$ to 7.8 months). Overall, adverse events (AEs) were clinically manageable and the most common were fatigue ( $9.9 \%$ of all AEs), paresthesia ( $6.5 \%$ of all AEs) and neutropenia (6.2\% of all AEs). Moreover, $49.5 \%$ of AEs were related to eribulin, $7.7 \%$ to bevacizumab, and $11.8 \%$ to both drugs. Quality of life was well preserved in most patients.

Conclusions: The results of this study suggest that continuing bevacizumab in combination with eribulin, beyond first line treatment with bevacizumab and paclitaxel is a reasonable therapeutic option for patients with HER2-negative MBC that exerts its effect without detrimentally affecting quality of life.

Trial registration: clinicalTrial.gov: NCT02175446. Registered 26, June 2014. https://clinicaltrials.gov/ct2/show/NCT02175446

\section{Background}

Metastatic breast cancer (MBC) continues to be incurable, however the development of novel therapeutic agents and recent advances in personalized treatment have considerably improved patient outcome [1]. Effective long-term management of MBC poses significant clinical challenges. The aim of current chemotherapeutic approaches is to improve survival duration and palliate symptoms while minimizing toxicity and maintaining quality of life [2]. The choice of MBC treatment is primarily influenced by the estrogen receptor (ER), the progesterone receptor (PR), and the human epidermal growth factor receptor 2 (HER2) status of the tumor [2]. Systemic chemotherapy is appropriate for women whose disease is refractory to endocrine therapy, is hormone receptor-negative or rapidly progressive with visceral 
involvement [2]. In the latter setting, combination chemotherapy is associated with a rapid response but greater toxicity and similar survival outcomes as the sequential use of single cytotoxic drugs [2]. The taxanes paclitaxel and docetaxel are a standard first-line option for MBC, but their use may be limited by primary or acquired resistance [3, 4]. In two large phase III trials [5, 6], progression-free survival (PFS) and response rates (RR) improved significantly when the anti-angiogenic VEGF-inhibitor bevacizumab was combined with paclitaxel or docetaxel as first-line therapy for locally recurrent or metastatic HER2negative breast cancer [7]. Moreover, in patients in the phase III TANIA trial whose HER2-negative breast cancer had progressed while on or after first line bevacizumab-containing therapy, further bevacizumab with second-line chemotherapy significantly improved PFS versus chemotherapy alone $[8,9]$.

Eribulin, a synthetic analog of the marine macrolide halichondrin $B$, is a microtubule inhibitor with a unique mechanism of action. Indeed, eribulin inhibits microtubule stability by blocking microtubule growth without affecting microtubule shortening, thereby sequestering b-tubulin into nonfunctional aggregates and leading to the formation of abnormal mitotic spindles and ultimately apoptosis [10, 11]. Recently, eribulin has been shown to exert also non-mitotic effects including remodeling of tumor vasculature with improved perfusion, reversal of the epithelial-to-mesenchymal transition, and decreased capacity for migration and invasion [12]. These effects, which in preclinical studies were found to reverse the aggressive characteristics of both the cellular phenotype and the microenvironment, may contribute to the clinical benefits of eribulin.

Several studies have demonstrated that eribulin is active in patients with MBC [13-16]. In the phase III EMBRACE trial, eribulin significantly improved overall survival (OS) versus the physician's treatment choice in patients with anthracyclines and taxane-pretreated locally recurrent or MBC [16]. Given the above results, we conducted a phase II, multicenter, single-arm trial (Gruppo Italiano Mammella [GIM]11BERGI) to evaluate the activity and safety of eribulin in combination with bevacizumab as second-line treatment for HER2-negative MBC progressing after first-line therapy with bevacizumab and paclitaxel.

\section{Methods}

Study design and patients. GIM11-BERGI is a phase II, multicenter, single-arm study following a Simon's two-stage optimal design [17]. All patients enrolled in the study received eribulin $1,23 \mathrm{mg} / \mathrm{m}^{2}$ on days 1 and 8 every 3 weeks intravenously combined with bevacizumab $15 \mathrm{mg} / \mathrm{kg}$ every 3 weeks or $10 \mathrm{mg} / \mathrm{kg}$ every 2 weeks intravenously, depending on the patient's or physician's preference (Figure 1). Study treatment was continued until disease progression, unacceptable toxicity, or patient withdrawal. If patients discontinued bevacizumab or eribulin for any reason before disease progression, the other treatment was continued as monotherapy until disease progression, unacceptable toxicity, or patient withdrawal. In the event of toxicity, neither dose reduction nor modification of bevacizumab was allowed, but bevacizumab was to be interrupted or permanently discontinued in case of hypertension, proteinuria, thrombosis, embolism, hemorrhage, congestive heart failure or wound-healing complications. 
Eligible women were those aged 18 years or older who had an HER2-negative breast cancer with documented progression of disease after or during first-line chemotherapy-based treatment with paclitaxel combined with bevacizumab for metastatic disease. Patients must have had a measurable disease per RECIST v1.1 criteria [18] according to investigator assessment. Patients with hormone receptor (HR)-positive disease may have been treated with one or more lines of endocrine-based therapy before receiving paclitaxel or bevacizumab. In addition, as part of their first line maintenance treatment, patients may have received bevacizumab monotherapy, bevacizumab plus endocrine treatment or no treatment (for $\leq 6$ weeks from the last bevacizumab administration).

All eligible patients must have an Eastern Cooperative Oncology Group (ECOG) performance status of 02 and an estimated life expectancy of 12 weeks or more. Patients were ineligible if they had previously received (i) first-line anti-angiogenic therapy (e.g. tyrosine kinase inhibitors [TKIs] or anti-vascular endothelial growth factors [anti-VEGFs]) other than bevacizumab for the first-line treatment of metastatic breast cancer; (ii) if they had exclusively received endocrine therapy combined with bevacizumab for the first-line treatment of metastatic breast cancer; positive or unknown HER2 status; (iii) inadequately controlled hypertension (defined as systolic blood pressure $>150 \mathrm{mmHg}$ and/or diastolic blood pressure $>100 \mathrm{mmHg}$ ) while receiving antihypertensive medication; (iv) a serious non-healing wound, active ulcer, or untreated bone fracture; (v) New York Heart Association Class II or greater congestive heart failure; pulmonary lymphangitis or pulmonary dysfunction requiring active treatment, including the use of oxygen; pre-existing grade $\geq 2$ neuropathy; (vi) serious active infection requiring intravenous antibiotics and/or hospitalization at study entry; (vii) history of hypertensive crisis, hypertensive encephalopathy, nephrotic syndrome, bleeding diathesis, clinically relevant coagulopathy, or grade 3 or 4 venous thromboembolism, and lastly (viii) a history of myocardial infarction, unstable angina, stroke or transient ischemic attack (TIA), significant vascular disease, gastrointestinal perforation, abdominal fistula, intraabdominal abscess, or active gastrointestinal bleeding within 6 months preceding study treatment. Patients were also ineligible if they had inadequate hematological function, coagulation parameters, hepatic function or renal function.

Statistical Analyses. A Simon's two-stage optimal design [17] was adopted to define the total number of patients required for this phase II study. An overall clinical response rate of $25 \%$ as the target activity level and $12 \%$ as the lowest response rate of interest were considered. The study was designed to have $80 \%$ power to accept the hypothesis and a $5 \%$ significance to reject the hypothesis. Therefore, the probability of accepting a therapy with a real response rate below $12 \%$ and the risk of rejecting a treatment with a response rate $25 \%$ would be, in both cases, less than $5 \%$.

At the first stage, and in order to proceed to stage II, 19 patients were monitored for a minimum of 18 weeks (corresponding to 6 cycles of study treatment). If there were fewer than 3 responses in the initial 19 patients, the study would have been stopped. Otherwise, 42 additional patients were planned to be accrued for a total of 61 patients. At the second stage, at least 12 objective responses in the 61 patients enrolled were required for this regimen to be deemed worthy of further investigation. At the second stage, patients were followed-up for a minimum of 18 weeks (corresponding to 6 cycles of study treatment). 
Outcomes. The primary study endpoint was the overall response rate (ORR) based on the best overall response as defined by RECIST criteria v. 1.1 (without confirmation). The best overall response rate (BORR) was estimated by the ratio of patients who had a complete response (CR) or partial response (PR) and the number of subjects in the intention-to-treat (ITT) population. The number of subjects with at least one post-baseline activity assessment was used as denominator in the per-protocol (PP) analysis in supportive analysis. The secondary efficacy variables were second-line progression-free survival (PFS), defined as the time from study enrollment to disease progression or death while on second-line treatment, and overall survival (OS) from enrollment to death from any cause, Clinical Benefit Rate (CBR) estimated by the ratio of patients who had a CR, PR or stable disease (SD) for $\geq 24$ weeks and the number of subjects in the ITT population, duration of response (DoR), safety and tolerability and Quality of Life (QoL). Second-line PFS and OS were estimated using the Kaplan Meier method. QoL was analyzed using a linear mixed model (random intercept only with time in months treated as continuous predictor) using all the available QoL assessments for each patient in the ITT population. Only data that were collected within the cutoff date (8, November 2017) were included in the present analyses.

Clinical examinations were carried out as clinically indicated before starting each cycle. Safety was assessed using the Common Terminology Criteria for Adverse Events (CTCAE) (version 4.0). All grade 35 adverse events and serious adverse events (any grade) were recorded at every study visit. All laboratory assessments were done locally according to local standards. All patients remained in the study with continued follow-up for OS, except those who withdrew consent, were lost to follow-up, or were removed from the study by the investigator (e.g., because of another illness, an adverse event, treatment failure after a prescribed procedure, protocol violation, cure, or due to administrative reasons). Patient-reported outcomes were assessed with the Functional Assessment of Cancer Therapy-Breast questionnaire (FACT-B).

\section{Results}

Between November 2014, and May 2016, eligible patients were enrolled at 16 sites in Italy. Of the 67 patients screened, 61 (91.0\%) were enrolled in the study (Figure 2). At data cut-off, 57 patients had discontinued therapy early (43 due to disease progression, 4 to withdrawn consent, 9 due to adverse events, one due to investigator's decision, and 4 had withdrawn consent). The remaining 4 patients continued to undergo the study treatment. All patients of the intention-to-treat (ITT) population were considered for the safety analysis. Three patients (4.5\%) were excluded from the per-protocol population because they had no post-baseline efficacy assessment. (Figure 2). The patients' baseline characteristics are reported in Table 1. Median age was 56.1 years; 50 patients (83.3\%) had hormonal receptor (estrogen receptor $[\mathrm{ER}]$ and/or progesterone $[\mathrm{PR}]$ ) positive (+) disease, and 10 patients $(16.7 \%)$ had triple-negative breast cancer (TNBC). All patients had previously received treatment with bevacizumab plus paclitaxel for a median duration of treatment of 9.77 months (range 2.43 to 34.20 months). At data cut-off, the median duration of follow-up was 16.2 months (range 1.6 to 26.7 months). 
Activity. In the first stage, three patients of the nineteen originally enrolled, achieved an objective response. The threshold for the first stage of Simon's two-stage design was reached and the trial continued to full accrual. Overall, the median duration of eribulin and bevacizumab treatment was 9.8 months (range 2.3 to 34.2 months). Objective responses were recorded in 58 of the 61 patients with measurable disease and at least one post-baseline tumor assessment. Among the 61 subjects of the ITT population, 1 patient $(1.6 \%$ ) had a complete response (CR), 14 patients $(23.0 \%)$ had a partial response (PR), and 26 patients (42.6\%) had best response of stable disease (SD) (Table 2). The BORR was $24.6 \%$ $(95 \% \mathrm{Cl}, 14.5 \%$ to $37.3 \%)$. Patients with SDER+ and ER- MBC achieved a similar rate of objective responses (Table 2). Twenty patients had a CR, PR or SD response that persisted for more than 24 weeks. The CBR was $32.8 \% \%(95 \% \mathrm{Cl}, 21.3 \%$ to $46.0 \%)$. In the subset of patients with objective responses $(\mathrm{n}=15)$, the median duration of response (DoR) was 6.2 months (range: 2.2 to 29.4 months).

At data cut-off of the analysis of second-line PFS, median follow-up was 16.2 months (range 1.6 to 26.7 months). The median PFS was 6.2 months ( $95 \% \mathrm{Cl}, 4.0$ to 7.8 ) (Figure 3A). No significant differences in median PFS between the ER+ and ER- subgroups were observed $(P=0.65)$ (Figure 3B). At data cut-off, fewer than half (2 of 61 ) of the patients had died, and, thus, median OS was not estimable. Quality of life was well preserved in most patients. In the efficacy analysis population, based on the longitudinal trajectories of FACT B total score, linear mixed model analysis showed a statistically significant, but clinically irrelevant, reduction of quality of life, with a 0.2-point reduction for every month of follow-up (95\% Cl, -0.28 to $-0.06, \mathrm{P}=0.004)$ (Figure 4).

Safety. Overall, $59 \%$ of patients experienced a treatment-related adverse event (AE). The most common AEs were fatigue, paresthesia, mucositis oral, and fever (Table 3). Grade 3 or worse AEs were observed in 23 of 61 patients (37.8\%), mainly owing to grade 3 hypertension (7\%), neutropenia (7\%) and febrile neutropenia (7\%). Adverse events resulted in the death of 2 patients (3.3\%) during treatment. In one case, death was not associated to any CTCAE term and was not related to the study drugs. In the other patient, death was associated to three concomitant SAE (i) hepatic failure, (ii) hypertransaminasemia and (iii) thrombocytopenia. All these concomitant SAEs were probably related to eribulin and were observed 177 days after treatment onset. Almost $50 \%$ of drug-related AEs were related to eribulin, $7.7 \%$ to bevacizumab, and $11.8 \%$ to both the study drugs. Treatment for AEs led to dose reductions in 15 patients (24.6\%), and were most often due to neutropenia and peripheral neuropathy. Four patients (6.6\%) discontinued eribulin treatment due to oral mucositis ( 2 patients), proteinuria (1 patient), and a thromboembolic event (1 patient).

\section{Discussion}

The results of the GIM11-BERGI trial showed that continuing bevacizumab in combination with eribulin beyond first line treatment with bevacizumab and paclitaxel is a reasonable therapeutic option for patients with HER2-negative MBC. The results of our "two step" Simon phase II trial confirm and expand 
the results of the TANIA $[8,9]$ and RIBBON-2 [19] trials in which the combination of bevacizumab with second-line chemotherapy improved PFS and ORR. In fact, the GIM11-BERGI median PFS of 6.3 months $(95 \% \mathrm{Cl}, 4.1$ to 7.8 months) and ORR of $24.6 \%$ are in line with the median PFS of 6.3 months $(95 \% \mathrm{Cl}, 0.61$ to 0.93 ) and an ORR of $21 \%$ reported in the TANIA trial, and similar to those observed in the RIBBON-2 trial (median PFS 7.2 months [95\% Cl, 0.64 to 0.93 ], ORR 39.5\%), albeit with the caveat of cross-trial comparisons. Notably, GIM11-BERGI is the first study to evaluate the efficacy and safety of the combination of eribulin and bevacizumab. Indeed, in the TANIA and RIBBON-2 studies, bevacizumab was administered in combination with capecitabine, taxanes and anthracyclines, but not eribulin.

Eribulin is currently a standard chemotherapy regimen for anthracycline- and taxane-pretreated metastatic breast cancer. In the phase III EMBRACE trial, eribulin induced a significant and clinically meaningful improvement in overall survival compared with the physician's choice of treatment in women with heavily metastatic breast cancer $(\mathrm{HR} 0.81 ; 95 \% \mathrm{Cl}, 0.66-0.99 ; \mathrm{P}=0.041)$ [16]. Eribulin also induced a numerical but not significant survival benefit compared with capecitabine in an earlier phase III trial [20]. Despite these positive results, the RR and median PFS observed in patients treated with eribulin in these two trials were modest, i.e., between $11-12 \%$ and 3.7-4.1 months, respectively. In this context, the encouraging results of our trial suggest that the combination of bevacizumab and eribulin may provide a valid treatment option after failure of first-line chemotherapy, that warrants validation in larger prospective trials.

Our results and those of the TANIA trial [8,9] support the importance of continued VEGF inhibition across treatment lines. Continued target inhibition is a strategy used in HER2-positive MBC, supported by results of the GBG 26 study [21] in which it was shown that continuing trastuzumab beyond progression is a safe and effective treatment approach.

Our results and those of the TANIA trial support the importance of continuous VEGF inhibition across treatments lines. This strategy, which is used in HER2-positive MBC [2], is supported by results of the GBG 26 study, which showed that continuing trastuzumab beyond disease progression is a safe and effective treatment approach $[21,22]$. They are also consistent with the finding of Bennua et al [23] that maintenance of VEGF inhibition with bevacizumab plus standard second-line chemotherapy beyond disease progression is associated with an improvement of median OS ( $\mathrm{HR}=0.81,95 \% \mathrm{Cl} 0.69-0.94$; $\mathrm{p}=0.0062)$ in patients with metastatic colorectal cancer.

The combination of bevacizumab with eribulin did not modify the safety profiles of either agent and did not cause fatal toxicities. The safety profile of bevacizumab is coherent with that observed in previous studies: hypertension was reported in $17 \%$ of patients ( $9 / 55$ pts) and proteinuria in $17 \%$ of cases $(9 / 55$ pts), while there was only one case of grade IV pulmonary arterial thromboembolism. No new side effects were observed with-long term bevacizumab treatment. Finally, in line with this favorable toxicity profile, the overall quality of life was well preserved in most patients enrolled in the study.

An additional aspect to consider regarding bevacizumb treatment is its high cost which has contributed to a cost-effectiveness controversy and brought into question its use in metastatic breast cancer. In 
November 2011, the United States Food and Drug Administration revoked its indication for bevacizumab for breast cancer patients. In contrast, European Medicines Association confirmed the combination of bevacizumab plus paclitaxel as a first-line treatment option for patients with HER2-negative metastatic breast cancer. Notably, the recent introduction into clinical practice of less costly bevacizumab biosimilars, which may result in a wider use of use of this agent.

We acknowledge that, similar to the TANIA trial, our study may have a selection bias, because we enrolled patients known to well tolerate bevacizumab. However, hypertension and proteinuria have been reported also in patients after long-term treatment ( $>1$ year) with bevacizumab, despite the absence of these toxicities in earlier administrations [24] [25]. In our experience, monitoring and management of these side effects could prevent treatment suspension, and their progression. We also acknowledge that in our study patients with hormonal receptor-positive breast cancer did not received CDK4/6 inhibitors in combination with endocrine therapy before study enrollment. CDK4/6 inhibitors are standard first- and second-line treatments for HR+/HER2-MBC [2], however, these agents were not available in clinical practice at the time of trial enrollment. Despite this limitation, our findings may still be clinically relevant especially for patients with HR+/HER2-negative MBC who do not benefit or progress after multiple lines of endocrinebased therapies, including the combinations with CDK4/6 inhibitors, and are thus candidates for chemotherapy.

\section{Conclusion}

Our results extend the finding that continuing bevacizumab beyond disease progression in association with eribulin could be a valid and well-tolerated treatment choice for HER2-negative MBC patients progressing after first-line bevacizumab with chemotherapy. Further comparative studies to verify the clinical benefits of this combination are needed.

\section{Declarations}

\section{Funding}

No specific funds were used for the conduction of this study nor for the writing of this paper.

\section{Authors' contributions}

All authors of this research paper have directly participated in the planning, execution, or analysis of the study. All authors were involved in manuscript writing and reviewing, gave their final approval and agreed to be accountable for all aspects of the work.

\section{Consent for publication}

Not applicable.

\section{Availability of data and materials}


The datasets during and/or analyzed during the current study available from the corresponding author on reasonable request.

\section{Ethics approval and consent to participate}

This research has been conducted in accordance with the Declaration of Helsinki. The study protocol and all modifications were approved by independent ethics committees at each participating site. All patients signed the informed consent form.

\section{Competing interests}

CDA is a consultant/advisory board member for Novartis, Eli Lilly, and Pfizer. FP has received travel, accommodations, expenses supported by Takeda, Ely Lilly, and received honoraria from Merck Sharp \& Dohme, Ely Lilly, Novartis outside the submitted work. AM is a consultant/advisory board member for EISAI, Novartis, Astra Zeneca, Teva, Pfizer, Celgene; he has received travel accommodations supported by Eisai, Celgene, Novartis, and Ipsen. LM is a consultant/advisory board member for Roche, Novartis, Celgene, Pfeizer, MSD, Genomic Health, Ipsen, Takeda, Eli Lilly, Seattle Genetics, Pierre Fabre, and Eisai. MG, GA and SDP have declared honoraria from Roche, Pfizer, Astra-Zeneca, Novartis, Celgene, Eli Lilly, Amgen and Eisai. MDL has declared consulting fees from Pfizer, Novartis, Eli Lilly, Roche, Eisai, and Celgene. The remaining authors declare that they have no conflicts of interest.

\section{Acknowledgments}

We gratefully thank Jean Ann Gilder (Scientific Communication srl., Naples, Italy) for editing the article and Oncotech for the data management of GIM11-BERGI Study.

\section{References}

1. Savard M-F, Khan O, Hunt KK, Verma S. Redrawing the Lines: The Next Generation of Treatment in Metastatic Breast Cancer. Am Soc Clin Oncol Educ book Am Soc Clin Oncol Annu Meet [Internet]. 2019;39:e8-21. Available from: http://www.ncbi.nlm.nih.gov/pubmed/31099662.

2. Cardoso F, Senkus E, Costa A, Papadopoulos E, Aapro M, André F, et al. 4th ESO-ESMO International Consensus Guidelines for Advanced Breast Cancer (ABC 4)十. Ann Oncol Off J Eur Soc Med Oncol [Internet]. 2018;29:1634-57. Available from: http://www.ncbi.nlm.nih.gov/pubmed/30032243.

3. Perez EA. Impact, mechanisms, and novel chemotherapy strategies for overcoming resistance to anthracyclines and taxanes in metastatic breast cancer. Breast Cancer Res Treat [Internet]. 2009;114:195-201. Available from: http://www.ncbi.nlm.nih.gov/pubmed/18443902.

4. Murray S, Briasoulis E, Linardou H, Bafaloukos D, Papadimitriou C. Taxane resistance in breast cancer: mechanisms, predictive biomarkers and circumvention strategies. Cancer Treat Rev [Internet]. 2012;38:890-903. Available from: http://www.ncbi.nlm.nih.gov/pubmed/22465195. 
5. Miles DW, Chan A, Dirix LY, Cortés J, Pivot X, Tomczak P, et al. Phase III study of bevacizumab plus docetaxel compared with placebo plus docetaxel for the first-line treatment of human epidermal growth factor receptor 2-negative metastatic breast cancer. J Clin Oncol [Internet]. 2010;28:3239-47. Available from: http://www.ncbi.nlm.nih.gov/pubmed/20498403.

6. Miller K, Wang M, Gralow J, Dickler M, Cobleigh M, Perez EA, et al. Paclitaxel plus bevacizumab versus paclitaxel alone for metastatic breast cancer. N Engl J Med [Internet]. 2007;357:2666-76. Available from: http://www.ncbi.nlm.nih.gov/pubmed/18160686.

7. Ferrara N, Hillan KJ, Gerber H-P, Novotny W. Discovery and development of bevacizumab, an antiVEGF antibody for treating cancer. Nat Rev Drug Discov [Internet]. 2004;3:391-400. Available from: http://www.ncbi.nlm.nih.gov/pubmed/15136787.

8. von Minckwitz G, Puglisi F, Cortes J, Vrdoljak E, Marschner N, Zielinski C, et al. Bevacizumab plus chemotherapy versus chemotherapy alone as second-line treatment for patients with HER2-negative locally recurrent or metastatic breast cancer after first-line treatment with bevacizumab plus chemotherapy (TANIA): an open-label, randomised. Lancet Oncol [Internet]. 2014;15:1269-78. Available from: http://www.ncbi.nlm.nih.gov/pubmed/25273342.

9. Vrdoljak E, Marschner N, Zielinski C, Gligorov J, Cortes J, Puglisi F, et al. Final results of the TANIA randomised phase III trial of bevacizumab after progression on first-line bevacizumab therapy for HER2-negative locally recurrent/metastatic breast cancer. Ann Oncol Off J Eur Soc Med Oncol [Internet]. 2016;27:2046-52. Available from: http://www.ncbi.nlm.nih.gov/pubmed/27502725.

10. Cigler T, Vahdat LT. Eribulin mesylate for the treatment of breast cancer. Expert Opin Pharmacother [Internet]. 2010;11:1587-93. Available from: http://www.ncbi.nlm.nih.gov/pubmed/20450446.

11. Kuznetsov G, Towle MJ, Cheng H, Kawamura T, TenDyke K, Liu D, et al. Induction of morphological and biochemical apoptosis following prolonged mitotic blockage by halichondrin $\mathrm{B}$ macrocyclic ketone analog E7389. Cancer Res [Internet]. 2004;64:5760-6. Available from: http://www.ncbi.nlm.nih.gov/pubmed/15313917.

12. Cortes J, Schöffski P, Littlefield BA. Multiple modes of action of eribulin mesylate: Emerging data and clinical implications. Cancer Treat Rev [Internet]. 2018;70:190-8. Available from: http://www.ncbi.nlm.nih.gov/pubmed/30243063.

13. Ortega V, Antón A, Garau I, Afonso N, Calvo L, Fernández Y, et al. Phase II, Multicenter, Single-arm Trial of Eribulin as First-line Therapy for Patients With Aggressive Taxane-pretreated HER2-Negative Metastatic Breast Cancer: The MERIBEL Study. Clin Breast Cancer [Internet]. 2019;19:105-12. Available from: http://www.ncbi.nlm.nih.gov/pubmed/30679100.

14. Vahdat LT, Pruitt B, Fabian CJ, Rivera RR, Smith DA, Tan-Chiu E, et al. Phase II study of eribulin mesylate, a halichondrin $B$ analog, in patients with metastatic breast cancer previously treated with an anthracycline and a taxane. J Clin Oncol [Internet]. 2009;27:2954-61. Available from: http://www.ncbi.nlm.nih.gov/pubmed/19349550.

15. Cortes J, Vahdat L, Blum JL, Twelves C, Campone M, Roché $H$, et al. Phase Il study of the halichondrin $B$ analog eribulin mesylate in patients with locally advanced or metastatic breast cancer 
previously treated with an anthracycline, a taxane, and capecitabine. J Clin Oncol [Internet]. 2010;28:3922-8. Available from: http://www.ncbi.nlm.nih.gov/pubmed/20679609.

16. Cortes J, O'Shaughnessy J, Loesch D, Blum JL, Vahdat LT, Petrakova K, et al. Eribulin monotherapy versus treatment of physician's choice in patients with metastatic breast cancer (EMBRACE): a phase 3 open-label randomised study. Lancet (London, England) [Internet]. 2011;377:914-23. Available from: http://www.ncbi.nlm.nih.gov/pubmed/21376385.

17. Simon R. Optimal two-stage designs for phase II clinical trials. Control Clin Trials [Internet]. 1989;10:1-10. Available from: http://www.ncbi.nlm.nih.gov/pubmed/2702835.

18. Schwartz LH, Litière S, de Vries E, Ford R, Gwyther S, Mandrekar S, et al. RECIST 1.1-Update and clarification: From the RECIST committee. Eur J Cancer [Internet]. 2016;62:132-7. Available from: http://www.ncbi.nlm.nih.gov/pubmed/27189322.

19. Brufsky AM, Hurvitz S, Perez E, Swamy R, Valero V, O'Neill V, et al. RIBBON-2: a randomized, doubleblind, placebo-controlled, phase III trial evaluating the efficacy and safety of bevacizumab in combination with chemotherapy for second-line treatment of human epidermal growth factor receptor 2-negative metastatic breast c. J Clin Oncol [Internet]. 2011;29:4286-93. Available from: http://www.ncbi.nlm.nih.gov/pubmed/21990397.

20. Kaufman PA, Awada A, Twelves C, Yelle L, Perez EA, Velikova G, et al. Phase III open-label randomized study of eribulin mesylate versus capecitabine in patients with locally advanced or metastatic breast cancer previously treated with an anthracycline and a taxane. J Clin Oncol [Internet]. 2015;33:594-601. Available from: http://www.ncbi.nlm.nih.gov/pubmed/25605862.

21. von Minckwitz G, Schwedler K, Schmidt M, Barinoff J, Mundhenke C, Cufer T, et al. Trastuzumab beyond progression: overall survival analysis of the GBG 26/BIG 3-05 phase III study in HER2positive breast cancer. Eur J Cancer [Internet]. 2011;47:2273-81. Available from: http://www.ncbi.nlm.nih.gov/pubmed/21741829.

22. von Minckwitz G, du Bois A, Schmidt M, Maass N, Cufer T, de Jongh FE, et al. Trastuzumab beyond progression in human epidermal growth factor receptor 2-positive advanced breast cancer: a german breast group 26/breast international group 03-05 study. J Clin Oncol [Internet]. 2009;27:1999-2006. Available from: http://www.ncbi.nlm.nih.gov/pubmed/19289619.

23. Bennouna J, Sastre J, Arnold D, Österlund P, Greil R, Van Cutsem E, et al. Continuation of bevacizumab after first progression in metastatic colorectal cancer (ML18147): a randomised phase 3 trial. Lancet Oncol [Internet]. 2013;14:29-37. Available from:

http://www.ncbi.nlm.nih.gov/pubmed/23168366.

24. Smith IE, Pierga J-Y, Biganzoli L, Cortés-Funes $H$, Thomssen C, Pivot $X$, et al. First-line bevacizumab plus taxane-based chemotherapy for locally recurrent or metastatic breast cancer: safety and efficacy in an open-label study in 2,251 patients. Ann Oncol Off J Eur Soc Med Oncol [Internet]. 2011;22:595-602. Available from: http://www.ncbi.nlm.nih.gov/pubmed/20819780.

25. Aogi K, Masuda N, Ohno S, Oda T, Iwata H, Kashiwaba M, et al. First-line bevacizumab in combination with weekly paclitaxel for metastatic breast cancer: efficacy and safety results from a 
large, open-label, single-arm Japanese study. Breast Cancer Res Treat [Internet]. 2011;129:829-38. Available from: http://www.ncbi.nlm.nih.gov/pubmed/21805309.

\section{Tables}

Table 1. Patients' characteristics 


\begin{tabular}{|c|c|}
\hline & ITT Population $(\mathrm{N}=61)$ \\
\hline Age & $56.1 \pm 11$ (34.1 to 78$)$ \\
\hline \multicolumn{2}{|c|}{ Hormonal receptor status (\%) } \\
\hline ER+ and/or PR+ & $50(83.3)$ \\
\hline ER- and PR- & $10(16.7 \%)$ \\
\hline missing & $1(1.6 \%)$ \\
\hline \multicolumn{2}{|c|}{ ECOG performance status } \\
\hline 0 & $56(91.8 \%)$ \\
\hline 1 & $5(8.2 \%)$ \\
\hline 2 & $0(0 \%)$ \\
\hline \multicolumn{2}{|c|}{ Number of metastatic sites } \\
\hline$<3$ & $32(52.5 \%)$ \\
\hline$\geq 3$ & $29(47.5 \%)$ \\
\hline \multicolumn{2}{|l|}{ Metastatic sites } \\
\hline Liver & $43(70.5 \%)$ \\
\hline Lung & $19(31.1 \%)$ \\
\hline Brain & $3(4.9 \%)$ \\
\hline Bone & $32(52.5 \%)$ \\
\hline Skin & $3(4.9 \%)$ \\
\hline Other & $34(55.7 \%)$ \\
\hline \multicolumn{2}{|l|}{ Site of disease } \\
\hline Visceral & $51(83.6 \%)$ \\
\hline Non-visceral & $10(16.4 \%)$ \\
\hline
\end{tabular}




\begin{tabular}{|l|l|}
\hline Prior endocrine therapy for advanced disease & \\
\hline Yes & $27(44.3 \%)$ \\
\hline No & $34(55.7 \%)$ \\
\hline
\end{tabular}

Table 2. Summary of treatment best responses.

\begin{tabular}{|c|c|c|c|c|c|}
\hline Outcome & $\begin{array}{l}\text { ITT } \\
\text { population } \\
(n=61)\end{array}$ & $\begin{array}{l}\text { PP population* } \\
(n=58)\end{array}$ & $\begin{array}{l}\text { ER+ } \\
(n=44)\end{array}$ & $\begin{array}{l}\text { ER- } \\
(n=13)\end{array}$ & $\begin{array}{l}\text { ER+ vs. } \\
\text { ER- } \\
\text { P-value }\end{array}$ \\
\hline BORR & 15 (24.6\%) & $\begin{array}{l}15(25.9 \% ; 95 \% \text { Cl } 15.3 \% \text { - } \\
39.0 \%)\end{array}$ & $\begin{array}{l}11 \\
(25.0 \%)\end{array}$ & $\begin{array}{l}4 \\
(30.8 \%)\end{array}$ & 0.727 \\
\hline CR & $1(1.6 \%)$ & $1(1.7 \%)$ & $0(0)$ & $1(7.7)$ & \multirow[t]{4}{*}{0.443} \\
\hline PR & $14(23.0 \%)$ & $14(17.2 \%)$ & $11(25)$ & $3(23.1)$ & \\
\hline SD & $26(42.6 \%)$ & $26(44.8 \%)$ & $20(45.5)$ & $6(46.2)$ & \\
\hline PD & $17(27.9 \%)$ & 17 (29.3\%) & $13(29.5)$ & $3(23.1)$ & \\
\hline
\end{tabular}

*Three patients without post-baseline efficacy assessments were excluded. ITT, intention-to-treat; PP, per-protocol.

Table 3. List of adverse events occurring at any grade in more than $5 \%$ of patients or grade $\geq 3$ in more than $2 \%$ of patients. 


\begin{tabular}{|llllll|}
\hline Adverse events & Grade 1 & Grade 2 & Grade 3 & Grade 4 & Grade 5 \\
\hline Fatigue & $17(28 \%)$ & $12(18 \%)$ & $3(5 \%)$ & 0 & 0 \\
\hline Paresthesia & $8(13 \%)$ & $10(16 \%)$ & $3(5 \%)$ & 0 & 0 \\
\hline Mucositis oral & $8(13 \%)$ & $7(11 \%)$ & $3(5 \%)$ & 0 & 0 \\
\hline Fever & $10(16 \%)$ & $6(10 \%)$ & $1(2 \%)$ & 0 & 0 \\
\hline Alopecia & $5(8 \%)$ & $3(5 \%)$ & $2(3 \%)$ & 0 & 0 \\
\hline Nausea & $12(20 \%)$ & $5(8 \%)$ & 0 & 0 & 0 \\
\hline Neutropenia & $5(8 \%)$ & $11(18 \%)$ & $4(7 \%)$ & 0 & 0 \\
\hline Constipation & $5(8 \%)$ & $4(7 \%)$ & $1(2 \%)$ & 0 & 0 \\
\hline Increased ALT/AST & $8(13 \%)$ & $1(2 \%)$ & 0 & $1(2 \%)$ & 0 \\
\hline Hypertension & $2(3 \%)$ & $3(5 \%)$ & $4(7 \%)$ & 0 & 0 \\
\hline Diarrhea & $6(10 \%)$ & 0 & 0 & 0 & 0 \\
\hline Headache & $5(8 \%)$ & $2(3 \%)$ & $1(2 \%)$ & 0 & 0 \\
\hline Peripheral sensory neuropathy & $8(13 \%)$ & $1(2 \%)$ & 0 & 0 & 0 \\
\hline Febrile neutropenia & 0 & $1(2 \%)$ & $4(7 \%)$ & 0 & 0 \\
\hline Cough & $3(5 \%)$ & $1(2 \%)$ & 0 & 0 & 0 \\
\hline Proteinuria & $3(5 \%)$ & $5(8 \%)$ & 0 & 0 & 0 \\
\hline Thrombocytopenia & $2(3 \%)$ & $4(7 \%)$ & 0 & 0 & $1(2 \%)$ \\
\hline Abdominal pain & $2(3 \%)$ & $2(3 \%)$ & $2(3 \%)$ & 0 & 0 \\
\hline Thromboembolic event & 0 & $1(2 \%)$ & 0 & $2(3 \%)$ & 0 \\
\hline
\end{tabular}

\section{Figures}



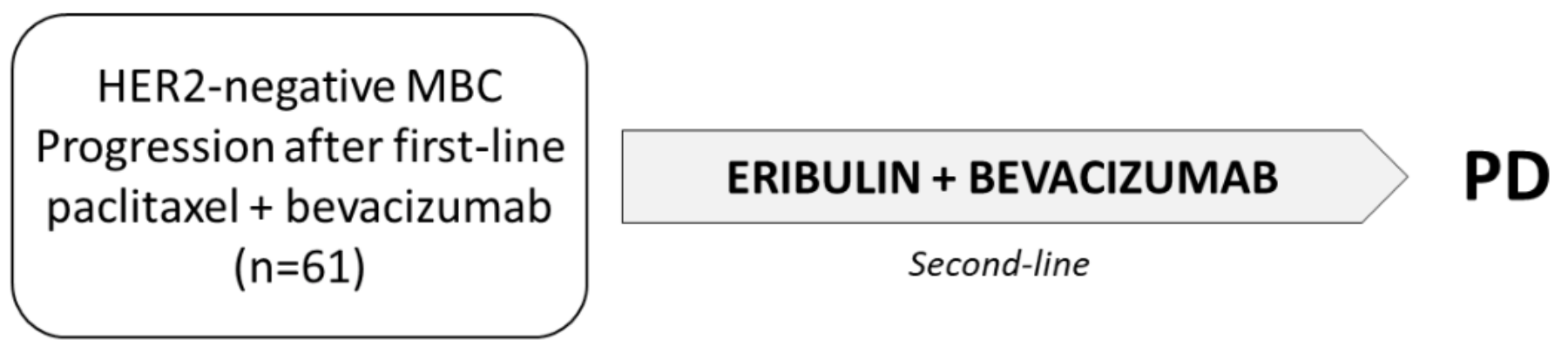

- Eribulin $1.23 \mathrm{mg} / \mathrm{m}^{2}$ on days 1, 8 every 3 weeks intravenously

- Bevacizumab $15 \mathrm{mg} / \mathrm{kg}$ every 3 weeks or $10 \mathrm{mg} / \mathrm{kg}$ every 2 weeks intravenously

Figure 1

Study design 


\section{7 patients screened}

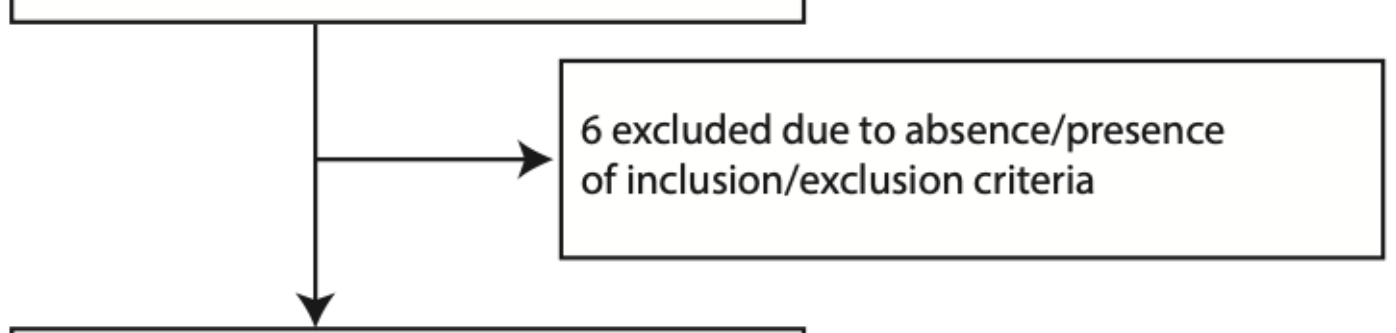

61 patients enrolled

57 discontinued intervention at data cutoff

43 disease progression

4 withdrew consent

9 adverse event ( 2 deaths)

1 medical decision

4 treatment ongoing at data cutoff

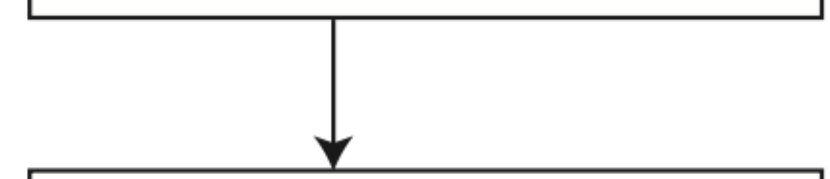

61 included in the ITT population

61 included in the Safety population

58 included in per-protocol population $†$

Figure 2

Trial profile 
A.

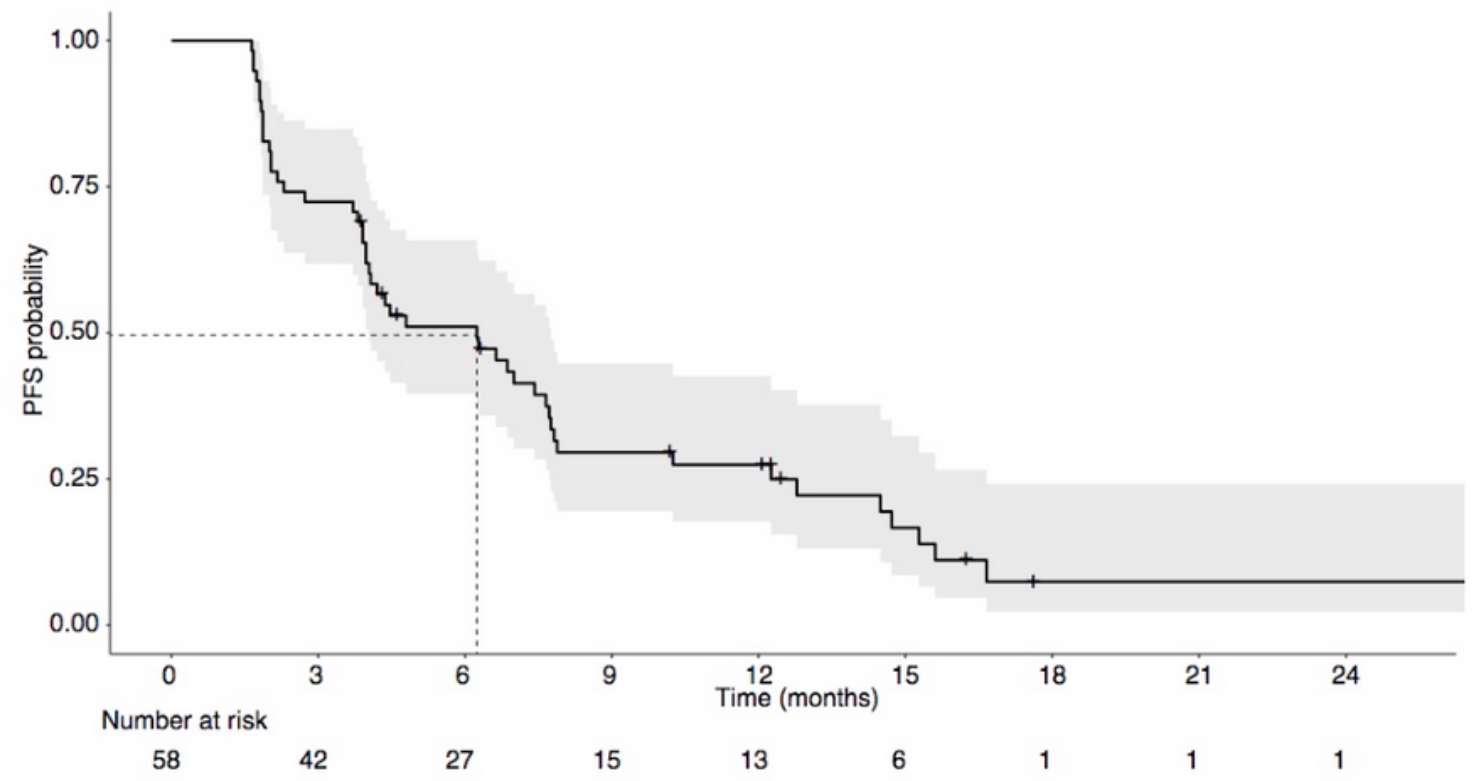

B.

Strata + ER- + ER+

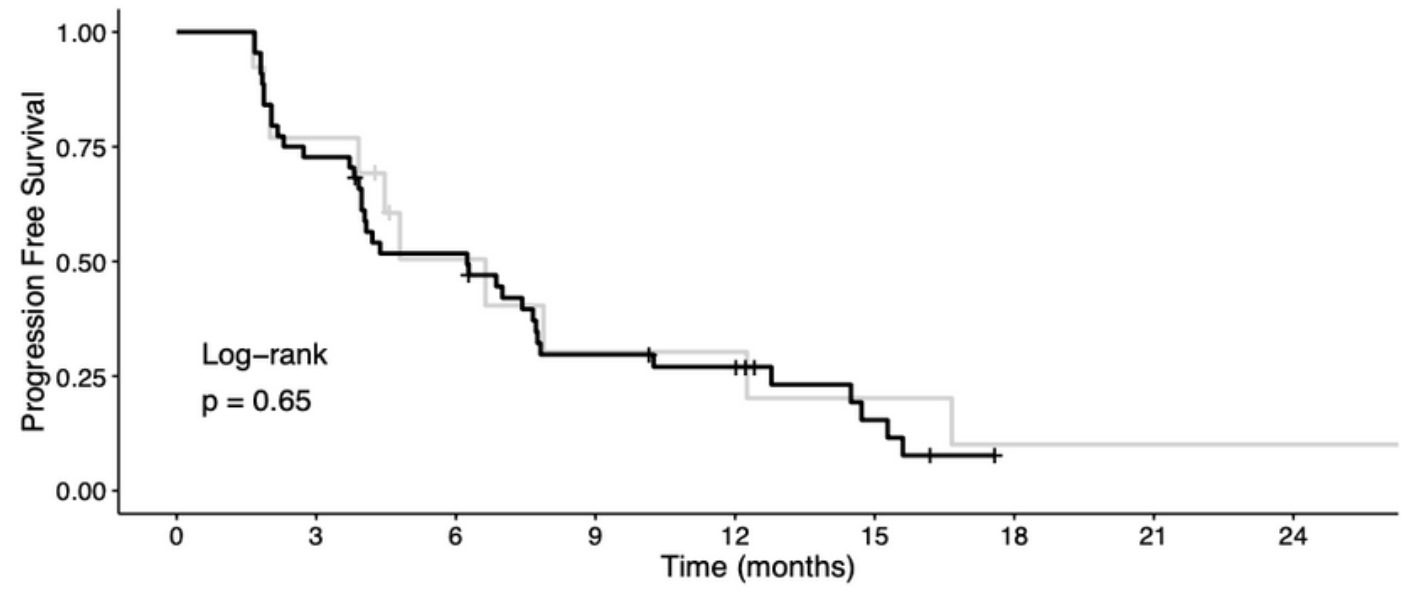

Number at risk

\begin{tabular}{|c|c|c|c|c|c|c|c|c|c|}
\hline \multirow{3}{*}{ 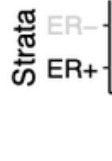 } & 13 & 10 & 5 & 3 & 3 & 2 & 1 & 1 & 1 \\
\hline & 44 & 32 & 22 & 12 & 10 & 4 & 0 & 0 & 0 \\
\hline & 0 & 3 & 6 & 9 & $\begin{array}{l}12 \\
0 \text { (n }\end{array}$ & 15 & 18 & 21 & 24 \\
\hline
\end{tabular}

\section{Figure 3}

Kaplan-Meier curves for progression-free survival (PFS) in all patients with at least one post-baseline efficacy assessment (A) and in the ER+ and ER-subgroups. 


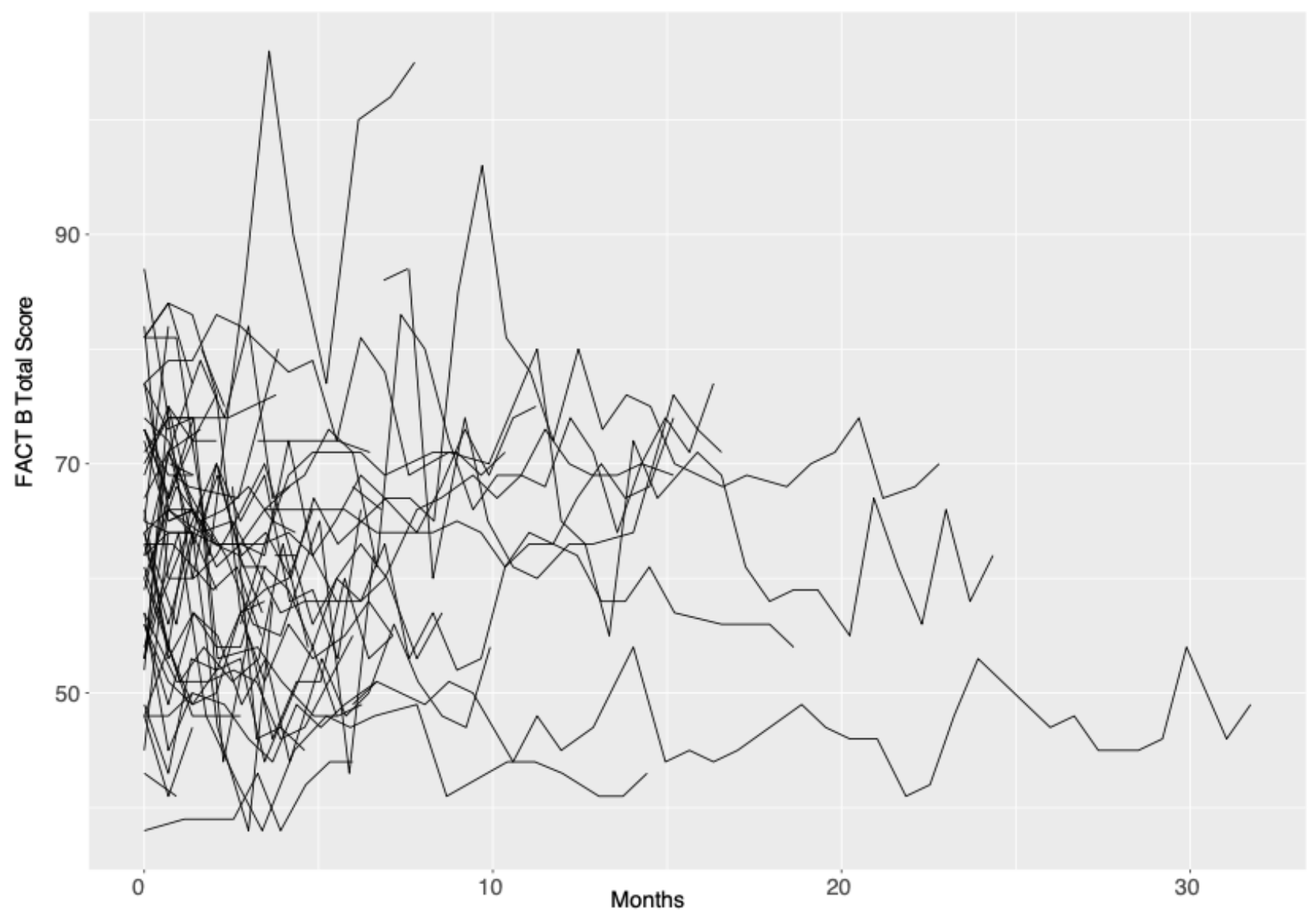

Figure 4

Individual trajectories of FACT B total score for all patients. 